\title{
Occurrence of Multiple Genomovars of Burkholderia cepacia in Cystic Fibrosis Patients and Proposal of Burkholderia multivorans sp. nov.
}

\author{
P. VANDAMME ${ }^{1,2 *}$ B. HOLMES, ${ }^{3}$ M. VANCANNEYT,${ }^{1}$ T. COENYE, ${ }^{1}$ B. HOSTE, ${ }^{1}$ R. COOPMAN, ${ }^{1}$ \\ H. REVETS, ${ }^{4}$ S. LAUWERS, ${ }^{5}$ M. GILLIS, ${ }^{1}$ K. KERSTERS, ${ }^{1}$ AND J. R. W. GOVAN ${ }^{6}$ \\ Laboratory of Microbiology, Universiteit Gent, Ghent, ${ }^{1}$ Department of Medical Microbiology, University Hospital UIA, \\ Antwerp, ${ }^{2}$ Laboratory of Cellular Immunology, Vlaams Instituut voor Biotechnologie, Vrije Universiteit Brussel, Sint- \\ Genesius-Rode, ${ }^{4}$ and Department of Microbiology, Academisch Ziekenhuis, Vrije Universiteit Brussel, Brussels, ${ }^{5}$ \\ Belgium, and National Collection of Type Cultures, Central Public Health Laboratory, London NW9 $5 H T,{ }^{3}$ and \\ Department of Medical Microbiology, University of Edinburgh Medical School, Edinburgh, ${ }^{6}$ United Kingdom
}

\begin{abstract}
We performed an integrated genotypic and phenotypic analysis of 128 strains of the genera Burkholderia, Ralstonia, and Pseudomonas in order to study the taxonomic structure of Burkholderia cepacia and its relationships with other Burkholderia species. Our data show that presumed $B$. cepacia strains isolated from cystic fibrosis patients belong to at least five distinct genomic species, one of which was identified as Burkholderia vietnamiensis. This group of five phenotypically similar species is referred to as the $B$. cepacia complex. The name Burkholderia multivorans is proposed for one of these genomic species, which was formerly referred to as B. cepacia genomovar II; the remaining B. cepacia groups are referred to as genomovars I, III, and IV, pending additional differential phenotypic tests. The role and pathogenic potential of each of these taxa, particularly in view of the potentially fatal infections in cystic fibrosis patients, need further evaluation. The data presented also demonstrate that Pseudomonas glathei and Pseudomonas pyrrocinia should be reclassified as Burkholderia species.
\end{abstract}

The taxonomy and the description of the genus Pseudomonas have been changing for more than a century (16). The discovery of five major phylogenetic lineages has played a major role (23) and was at the origin of the description of several new genera, the authentic pseudomonads being confined to those species belonging to the same lineage as Pseudomonas aeruginosa, the type species $(9,23)$. Yabuuchi et al. (43) reclassified a number of Pseudomonas species belonging to rRNA group II (21) as Burkholderia species. However, these authors examined only some of the species belonging to rRNA group II, and their conclusions were based on a limited set of strains. As a consequence, several additional Pseudomonas species had to be reclassified as Burkholderia species in subsequent studies $(10,35,46)$ and the genus Ralstonia was created to accommodate the generically misclassified species Burkholderia solanacearum and Burkholderia pickettii $(10,44)$. At present, the genus Burkholderia comprises 11 species: Burkholderia andropogonis, Burkholderia caryophylli, Burkholderia cepacia (the type species), Burkholderia cocovenenans, Burkholderia gladioli, Burkholderia glumae, Burkholderia mallei, Burkholderia plantarii, Burkholderia pseudomallei, Burkholderia vandii, and Burkholderia vietnamiensis (10).

During the last decade, B. cepacia, traditionally a plant pathogen occurring in diverse ecological niches, has caused great concern among cystic fibrosis patients and their caregivers. The narrow spectrum of bacteria causing severe pulmonary exacerbations in cystic fibrosis patients was traditionally confined to Staphylococcus aureus, Haemophilus influenzae, and $P$. aeruginosa (12). Since the early 1980 s, increasing numbers of $B$. cepacia infections have been reported in several

* Corresponding author. Mailing address: Laboratorium voor Microbiologie, Ledeganckstraat 35, B-9000 Ghent, Belgium. Phone: (32)9.264.51.13. Fax: (32)9.264.50.92. E-mail: Peter.Vandamme@rug ac.be. countries $(1,12)$. Infection or colonization by $B$. cepacia strains led to different outcomes in different patients. A major cause for concern was that about $20 \%$ of patients succumb to $B$. cepacia syndrome, a necrotizing pneumonia which culminates in rapid and fatal clinical deterioration $(12,15)$. Other patients are chronically colonized without, or with only a slow, deterioration of lung function $(11,26,28)$. In addition, several cases of $B$. gladioli infections or infections caused by organisms with characteristics seemingly intermediate between $B$. gladioli and B. cepacia were reported $(5,28)$.

The term genomovar was recently introduced to denote phenotypically similar but genotypically distinct groups of strains that were previously referred to by a variety of different terms including genomic species, genomic groups, genospecies, or genomospecies (36). These genomovars share a low level of DNA hybridization and therefore represent distinct species for which an official binomial name is not proposed pending the availability of differential diagnostic tests.

The present study was initiated by the receipt, at about the same time, of several strains taken from cystic fibrosis patients from two different centers in the English Midlands for computer-assisted identification at the National Collection of Type Cultures (NCTC). The strains were difficult to identify because they were phenotypically heterogeneous, with some being nonsaccharolytic while others were saccharolytic and closely resembled $B$. cepacia. Nevertheless, the isolates had virtually identical whole-cell protein patterns as determined by sodium dodecyl sulfate-polyacrylamide gel electrophoresis (SDSPAGE). As concern regarding $B$. cepacia increased (1) and laboratory identification improved, such strains were recognized from other parts of the United Kingdom and from Belgium during a national surveillance study (26). In addition, several studies revealed considerable heterogeneity among strains tentatively classified as $B$. cepacia $(2,3,10,28,33,34$, 
45). These initial observations prompted the fuller polyphasic study described herein.

\section{MATERIALS AND METHODS}

Bacterial strains and growth conditions. All strains studied and their sources are listed in Table 1. Thirty-nine reference strains were obtained from international culture collections. In addition, 89 recent isolates identified as $B$. cepacia by means of biochemical tests, cellular fatty acid analysis, or both were included. All reference strains and a selection of the field isolates examined (see below) are available from the Belgian Coordinated Collections of Microorganisms/Laboratorium Microbiologie Gent (BCCM/LMG) and NCTC culture collections.

Bacteriological purity was checked by plating, gram staining, and examining living cells by phase contrast microscopy. Strains were grown on nutrient agar containing $0.1 \%(\mathrm{wt} / \mathrm{vol})$ beef extract, $0.2 \%$ yeast extract, $0.5 \%(\mathrm{wt} / \mathrm{vol}) \mathrm{NaCl}$, $0.5 \%$ (wt/vol) peptone, $0.04 \%$ (wt/vol) $\mathrm{KH}_{2} \mathrm{PO}_{4}, 0.24 \%$ (wt/vol) $\mathrm{Na}_{2} \mathrm{HPO}_{4}$. $12 \mathrm{H}_{2} \mathrm{O}$, and $2.0 \%$ (wt/vol) agar ( $\mathrm{pH} 6.8$ ) and incubated aerobically at $28^{\circ} \mathrm{C}$ unless indicated otherwise.

PAGE of whole-cell proteins. After an incubation period of $48 \mathrm{~h}$, whole-cell protein extracts were prepared and SDS-PAGE was performed as described before (25). The densitometric analysis, normalization and interpolation of the protein profiles, and numerical analysis were performed by using the GelCompar software package, version 4.0 (Applied Maths, Kortrijk, Belgium).

Preparation of high-molecular-weight DNA. High-molecular-weight native DNA was prepared as described before (39). DNA was extracted from representative strains of all clusters or subclusters containing presumed $B$. cepacia strains: i, v, vi, vii, viii, $x, x i$, xiia, and xiib (see below). DNA was also prepared from $B$. cepacia LMG 14939 , which occupied a separate position in the dendrogram, and from the $B$. cocovenenans, $B$. gladioli, $P$. glathei, and $P$. pyrrocinia type strains.

DNA base compositions. All of the mean moles percent guanine plus cytosine values were determined by thermal denaturation and calculated as described by De Ley (7)

DNA-rRNA hybridization experiments. In vivo ${ }^{3} \mathrm{H}$-labelled $23 \mathrm{~S}$ rRNA from $B$. cepacia LMG $1222^{\mathrm{T}}$ was available from previous studies (10). Purification of rRNA fractions, fixation of single-stranded DNA on membrane filters, chemical determination of the amount of DNA on the filter, saturation hybridization, ribonuclease treatment, and thermostability measurement of the hybrids were performed as described by Van Landschoot and De Ley (40). Each DNA-rRNA hybrid is characterized by its $T_{m(e)}$ value (the melting temperature of elution), the temperature at which $50 \%$ of the DNA-rRNA hybrid is denatured. The higher the $T_{m(e)}$ of a heterologous hybrid, the closer both strains are related.

DNA-DNA hybridization experiments. The degree of DNA-DNA binding, expressed as a percent, was determined spectrophotometrically by the initial renaturation rate method of De Ley et al. (8). Each value given is the average of at least two hybridization experiments. Values of $30 \%$ DNA binding and less do not represent significant DNA homology. The total DNA concentration was 65 $\mu \mathrm{g} / \mathrm{ml}$, and the optimal renaturation temperature in $2 \times \mathrm{SSC}$ (sodium saline citrate; $1 \times \mathrm{SSC}$ is $0.15 \mathrm{M} \mathrm{NaCl}$ plus $0.015 \mathrm{M}$ sodium citrate, $\mathrm{pH} \mathrm{7}$ ) was $80.7^{\circ} \mathrm{C}$.

Fatty acid methyl ester analysis. After an incubation period of $24 \mathrm{~h}$ on Trypticase soy agar at $35^{\circ} \mathrm{C}$ (catalog no. 11768; BBL, Becton Dickinson Microbiology Systems, Cockeysville, Md.), a loopful of well-grown cells was harvested and fatty acid methyl esters were prepared, separated, and identified by using the Microbial Identification System (Microbial ID, Inc., Newark, Del.) as described before (39).

Conventional biochemical tests. All saccharolytic strains were characterized in a range of 68 conventional biochemical tests by methods described previously (14); nonsaccharolytic strains were characterized in a limited range of these tests.

\section{RESULTS}

PAGE of whole-cell proteins. Duplicate protein extracts were prepared to check the reproducibility of the growth conditions and the preparation of the extracts. The correlation level between duplicate protein patterns was more than $93 \%$.

The whole-cell protein patterns of several Burkholderia strains were often characterized by a distortion of part of the banding pattern. This "smiling" appearance is probably due to the presence of polysaccharides which are not adequately removed during the extraction procedure and was a reproducible phenomenon, as repeated extractions resulted in similar distortions. It was essential not to exceed the amount of 80 to 90 mg of wet cell weight for the extraction of the cellular proteins (see the procedure described by Pot et al. [25]). When present, distortions were mostly seen in the 45,000 - to 66,000 -molecular-weight region (data not shown) and influenced the correlation level between protein patterns. It was thus essential to thoroughly compare the clustering result of the numerical analysis of the protein patterns (Fig. 1) with the profiles themselves in order to significantly delineate clusters of strains with similar protein patterns.

After extensive examination of the protein profiles and the dendrogram, 12 clusters were delineated (Fig. 1). Several strains occupied a separate position in the dendrogram. Reference strains and recent isolates of $B$. cepacia constituted eight distinct clusters: $\mathrm{i}, \mathrm{v}, \mathrm{vi}$, vii, viii, $\mathrm{x}, \mathrm{xi}$, and xii.

Cluster i comprises 10 B. cepacia reference strains (including the type strain), the $P$. pyrrocinia type strain, a single cystic fibrosis isolate (LMG 14095), 6 strains from the hospital environment, 2 strains from non-cystic fibrosis patients, and 4 strains from plant rhizospheres or bark. $P$. pyrrocinia LMG $14191^{\mathrm{T}}$ and strain LMG 14086 group at the edge of this cluster. Cluster ii comprises two subclusters and the $B$. cocovenenans type strain, which occupies a separate position. Subclusters iia and iib comprise the three reference strains of $B$. gladioli and $B$. glumae, respectively. Cluster iii contains the $P$. aeruginosa reference strains. Cluster iv comprises the $B$. plantarii reference strains and $B$. vandii, which have indistinguishable protein patterns. Ralstonia eutropha LMG $1199^{\mathrm{T}}$, B. andropogonis LMG $2129^{\mathrm{T}}$, B. caryophylli LMG $2155^{\mathrm{T}}$, and cluster v (B. cepacia LMG 2161 and LMG 14087) group near cluster iv, but each occupies a separate position. Cluster vi is composed of five $B$. cepacia strains which were isolated from three Belgian cystic fibrosis patients. Cluster vii comprises a $B$. vietnamiensis reference strain (LMG 6998), two strains from Swedish cystic fibrosis patients which were originally identified as B. cepacia, and a soil isolate. Similarly, cluster viii comprises three $B$. vietnamiensis reference strains (including the type strain) and a single soil isolate originally identified as $B$. cepacia. In addition, $B$. vietnamiensis LMG 6999 occupies a separate position in the dendrogram. The two $P$. glathei reference strains constitute cluster ix. Cluster x comprises 15 B. cepacia strains. Twelve isolates were obtained from four cystic fibrosis patients (three from Belgium and one from the United Kingdom), one was isolated from a hospital flower vase, one from soil, and one from a non-cystic fibrosis patient. Cluster xi comprises four isolates obtained from three cystic fibrosis patients (one from Belgium and two from the United Kingdom). Cluster xii comprises $47 \mathrm{~B}$. cepacia isolates obtained from 41 cystic fibrosis patients ( 6 from Belgium and 35 from the United Kingdom) and two lung transplant patients (we do not know if the latter patients are cystic fibrosis patients or not). Three isolates from Belgium and one from the United Kingdom constitute a first subcluster (xiia), while the remaining strains constitute subcluster xiib. Strain B. cepacia LMG 14939 occupies a separate position near cluster xii. Finally, the Ralstonia pickettii and Ralstonia solanacearum type strains each occupy separate positions in the dendrogram.

DNA-rRNA hybridization experiments. The DNA-rRNA hybridization results are listed in Table 2. B. cepacia strains from clusters $\mathrm{i}, \mathrm{v}, \mathrm{vi}, \mathrm{x}, \mathrm{xi}$, and xii all exhibit high $T_{m(e)}$ values $\left(79.0\right.$ to $81.6^{\circ} \mathrm{C}$ ) versus $B$. cepacia LMG $1222^{\mathrm{T}}$ rRNA. $B$. cocovenenans LMG $11626^{\mathrm{T}}$ occupies a more distinct position, somewhat lower on the $B$. cepacia rRNA branch $\left(T_{m(e)}\right.$, $\left.77.7^{\circ} \mathrm{C}\right) . P$. pyrrocinia, and $P$. glathei reference strains also belong to the $B$. cepacia rRNA branch, with $T_{m(e)}$ values similar to those of $B$. caryophylli and $B$. andropogonis, respectively (Table 2).

DNA-DNA hybridization experiments. The results of the DNA-DNA hybridizations are summarized in Table 3. Within the group of $B$. cepacia strains examined, five major genotypic subgroups can be delineated. One of these genotypic subgroups was identified as $B$. vietnamiensis; the others are re- 
TABLE 1. List of strains studied

\begin{tabular}{|c|c|c|c|c|}
\hline $\begin{array}{l}\text { Name as received and strain } \\
\text { designation (revised name) })^{a}\end{array}$ & Genomovar & Type of analysis $^{b}$ & $\begin{array}{c}\text { Other strain } \\
\text { designation(s) }^{c}\end{array}$ & Source $^{d}$ \\
\hline B. andropogonis LMG $2129^{\mathrm{T}}$ & & $\mathrm{f}$ & NCPPB $934^{\mathrm{T}}$ & Sorghum bicolor \\
\hline B. caryophylli LMG $2155^{\mathrm{T}}$ & & $\mathrm{f}$ & NCPPB $2151^{\mathrm{T}}$ & Dianthus caryophyllus \\
\hline \multicolumn{5}{|l|}{ B. cepacia reference strains: } \\
\hline LMG $1222^{\mathrm{T}}$ & I & $\mathrm{i}, \mathrm{f}, \mathrm{b}$ & $\begin{array}{l}\text { Palleroni } 717^{\mathrm{T}} \\
\quad \text { NCTC } 10743^{\mathrm{T}}\end{array}$ & Allium cepa \\
\hline LMG 6863 & I & $i, f$ & NCIB 9690 & River Maraval (Trinidad) \\
\hline LMG 6963 & I & $\mathrm{i}, \mathrm{f}$ & PDDCC 5981 & Soil (Australia) \\
\hline LMG 6964 & I & $\mathrm{i}, \mathrm{f}, \mathrm{b}$ & PDDCC 5982 & Lycopersicum lycopersicum (L.) (Australia) \\
\hline LMG 6981 & I & $\mathrm{i}, \mathrm{f}, \mathrm{b}$ & $\begin{array}{l}\text { ATCC } 25609 \\
\text { NCTC } 10744\end{array}$ & Bronchial washing \\
\hline LMG 6986 & I & $\mathrm{i}, \mathrm{f}, \mathrm{b}$ & CCUG 788 & Urine \\
\hline LMG 6988 & I & $\mathrm{i}, \mathrm{f}, \mathrm{b}$ & CCUG 1603 & Leg wound (Sweden, 1972) \\
\hline LMG 6992 & I & $\mathrm{i}, \mathrm{f}, \mathrm{b}$ & CCUG 2857 & Soil (Trinidad, 1960) \\
\hline LMG 6997 & I & $i, f, b$ & CCUG 3461B & Ear (Sweden, 1974) \\
\hline LMG 7000 & I & $\mathrm{i}, \mathrm{f}, \mathrm{b}$ & CCUG 13348 & Blood (Sweden, 1983) \\
\hline LMG 2161 & I & $\mathrm{v}$ & NCTC 10661 & Forest soil (Trinidad) \\
\hline LMG 17588 (B. multivorans) & II & $\mathrm{x}$ & ATCC 17616 & Soil (United States) \\
\hline \multicolumn{5}{|l|}{ B. cepacia field isolates: } \\
\hline LMG 13014 & I & $\mathrm{i}, \mathrm{f}$ & & Hand cream (Belgium, 1991) \\
\hline LMG 13015 & I & $\mathrm{i}, \mathrm{f}$ & & Humidifier (Belgium, 1991) \\
\hline LMG 13016 & I & $\mathrm{i}, \mathrm{f}$ & & Catheter (Belgium, 1991) \\
\hline LMG 13017 & I & $\mathrm{i}, \mathrm{f}$ & & Blood culture (Belgium, 1988) \\
\hline LMG 13018 & I & $\mathrm{i}, \mathrm{f}$ & & Blood culture (Belgium, 1989) \\
\hline LMG 14086 & I & $\mathrm{i}, \mathrm{f}, \mathrm{b}$ & & Respirator (United Kingdom, 1970) \\
\hline LMG 14095 & I & $\mathrm{i}, \mathrm{f}, \mathrm{b}$ & & $\begin{array}{l}\text { Cystic fibrosis patient (United Kingdom, } \\
\text { 1974) }\end{array}$ \\
\hline LMG 15950 & I & $\mathrm{i}, \mathrm{f}$ & & $\begin{array}{l}\text { Water bath, cystic fibrosis ward (Belgium, } \\
\text { 1994) }\end{array}$ \\
\hline LMG 15951 & I & $\mathrm{i}, \mathrm{f}$ & & Spirometer (Belgium, 1994) \\
\hline LMG 16669 & I & $\mathrm{i}$ & $\mathrm{J} 2541$ & $\begin{array}{l}\text { Rhizosphere of Epidendrum o'bienianum } \\
\text { (United Kingdom) }\end{array}$ \\
\hline LMG 16670 & I & $\mathrm{i}$ & $\mathrm{J} 2552$ & $\begin{array}{l}\text { Rhizosphere of Carludovica palmata } \\
\text { (United Kingdom) }\end{array}$ \\
\hline LMG 16671 & I & $\mathrm{i}$ & $\mathrm{J} 2535$ & $\begin{array}{l}\text { Rotting bark, orchid house (United } \\
\text { Kingdom) }\end{array}$ \\
\hline LMG 16672 & I & $\mathrm{i}$ & $\mathrm{J} 2540$ & $\begin{array}{l}\text { Rhizosphere of banana plant (United } \\
\text { Kingdom) }\end{array}$ \\
\hline LMG 14087 & I & $\mathrm{v}, \mathrm{f}, \mathrm{b}$ & & Wound swab (United Kingdom, 1988) \\
\hline LMG $13010^{\mathrm{T}}$ (B. multivorans) & II & $\mathrm{x}, \mathrm{f}, \mathrm{b}$ & NCTC $13007^{\mathrm{T}}$ & Cystic fibrosis patient (Belgium, 1992) \\
\hline LMG 14273 (B. multivorans) & II & $x, f, b$ & & Cystic fibrosis patient (Belgium, 1993) \\
\hline LMG 14275 (B. multivorans) & II & $\mathrm{x}, \mathrm{f}$ & & Cystic fibrosis patient (Belgium, 1993) \\
\hline LMG 14277 (B. multivorans) & II & $\mathrm{x}, \mathrm{f}, \mathrm{b}$ & & Cystic fibrosis patient (Belgium, 1993) \\
\hline LMG 14280 (B. multivorans) & II & $x, f, b$ & & Cystic fibrosis patient (Belgium, 1993) \\
\hline LMG 14281 (B. multivorans) & II & $\mathrm{x}, \mathrm{f}, \mathrm{b}$ & & Cystic fibrosis patient (Belgium, 1993) \\
\hline LMG 14282 (B. multivorans) & II & $\mathrm{x}, \mathrm{f}, \mathrm{b}$ & & Cystic fibrosis patient (Belgium, 1993) \\
\hline LMG 14284 ( $B$. multivorans) & II & $\mathrm{x}, \mathrm{f}, \mathrm{b}$ & & Cystic fibrosis patient (Belgium, 1993) \\
\hline LMG 14286 (B. multivorans) & II & $x, f, b$ & & Cystic fibrosis patient (Belgium, 1993) \\
\hline LMG 14293 (B. multivorans) & II & $x, f, b$ & & Cystic fibrosis patient (Belgium, 1993) \\
\hline LMG 14296 (B. multivorans) & II & $x, f, b$ & & Cystic fibrosis patient (Belgium, 1993) \\
\hline LMG 16660 (B. multivorans) & II & $x, f$ & C1576 & $\begin{array}{l}\text { Cystic fibrosis patient (United Kingdom), } \\
\text { Glasgow epidemic reference }\end{array}$ \\
\hline LMG 16665 (B. multivorans) & II & $x, f$ & C1962 & $\begin{array}{l}\text { Non-cystic fibrosis patient, brain abscess } \\
\text { (United Kingdom) }\end{array}$ \\
\hline LMG 16668 (B. multivorans) & II & $\mathrm{x}, \mathrm{f}$ & $\mathrm{J} 2395$ & Hospital flower vase (United Kingdom) \\
\hline LMG 13053 & III & $\mathrm{xi}, \mathrm{f}$ & & Cystic fibrosis patient (Belgium, 1987) \\
\hline LMG 14274 & III & $x i, f$ & & Cystic fibrosis patient (Belgium, 1993) \\
\hline LMG 16661 & III & $x i, f$ & & Cystic fibrosis patient (United Kingdom) \\
\hline LMG 16662 & III & $\mathrm{xi}, \mathrm{f}$ & & Cystic fibrosis patient (United Kingdom) \\
\hline LMG 14270 & III & xiia, $f, b$ & & Cystic fibrosis patient (Belgium, 1993) \\
\hline LMG 14271 & III & xiia, $\mathrm{f}$ & & Cystic fibrosis patient (Belgium, 1993) \\
\hline LMG 14942 & III & xiia, $f, b$ & & Cystic fibrosis patient (Belgium, 1993) \\
\hline LMG 16659 & III & xiia & C1394 & $\begin{array}{l}\text { Cystic fibrosis patient (United Kingdom), } \\
\text { Manchester epidemic reference }\end{array}$ \\
\hline LMG 12614 & III & xiib, f, b & NCTC 13010 & $\begin{array}{l}\text { Cystic fibrosis patient (United Kingdom, } \\
\text { 1992) }\end{array}$ \\
\hline LMG 12615 & III & xiib, $f, b$ & NCTC 13008 & $\begin{array}{l}\text { Cystic fibrosis patient (United Kingdom, } \\
\text { 1992) }\end{array}$ \\
\hline
\end{tabular}


TABLE 1-Continued

\begin{tabular}{|c|c|c|c|c|}
\hline $\begin{array}{l}\text { Name as received and strain } \\
\text { designation (revised name) }\end{array}$ & Genomovar & Type of analysis ${ }^{b}$ & $\begin{array}{l}\text { Other strain } \\
\text { designation }(\mathrm{s})^{c}\end{array}$ & Source $^{d}$ \\
\hline LMG 13011 & III & xiib, $\mathrm{f}$ & & Cystic fibrosis patient (Belgium, 1988) \\
\hline LMG 13012 & III & xiib, $f$ & & Cystic fibrosis patient (Belgium, 1988) \\
\hline LMG 13054 & III & xiib, f & & Cystic fibrosis patient (Belgium, 1987) \\
\hline LMG 13307 & III & xiib, $\mathrm{f}, \mathrm{b}$ & ISL $866 / 92$ & $\begin{array}{l}\text { Cystic fibrosis patient (United Kingdom, } \\
\text { 1992) }\end{array}$ \\
\hline LMG 13308 & III & xiib, f, b & ISL $101 / 92$ & $\begin{array}{l}\text { Cystic fibrosis patient (United Kingdom, } \\
\text { 1992) }\end{array}$ \\
\hline LMG 13309 & III & xiib, f, b & ISL $464 / 92$ & $\begin{array}{l}\text { Cystic fibrosis patient (United Kingdom, } \\
\text { 1992) }\end{array}$ \\
\hline LMG 13310 & III & xiib, $f, b$ & ISL $1021 / 92$ & $\begin{array}{l}\text { Cystic fibrosis patient (United Kingdom, } \\
\text { 1992) }\end{array}$ \\
\hline LMG 13311 & III & xiib, $f, b$ & ISL $87 / 92$ & $\begin{array}{l}\text { Cystic fibrosis patient (United Kingdom, } \\
\text { 1992) }\end{array}$ \\
\hline LMG 13312 & III & xiib, $\mathrm{f}, \mathrm{b}$ & ISL $864 / 92$ & $\begin{array}{l}\text { Cystic fibrosis patient (United Kingdom, } \\
\text { 1992) }\end{array}$ \\
\hline LMG 13313 & III & xiib, $f, b$ & ISL $878 / 92$ & $\begin{array}{l}\text { Lung transplant patient (United Kingdom, } \\
\text { 1992) }\end{array}$ \\
\hline LMG 13314 & III & xiib, $f, b$ & ISL $7 / 92$ & $\begin{array}{l}\text { Cystic fibrosis patient (United Kingdom, } \\
\text { 1992) }\end{array}$ \\
\hline LMG 13315 & III & xiib, $f, b$ & ISL $854 / 92$ & $\begin{array}{l}\text { Cystic fibrosis patient (United Kingdom, } \\
\text { 1992) }\end{array}$ \\
\hline LMG 13316 & III & xiib, f, b & ISL $856 / 91$ & $\begin{array}{l}\text { Cystic fibrosis patient (United Kingdom, } \\
\text { 1991) }\end{array}$ \\
\hline LMG 13317 & III & xiib, f, b & ISL $865 / 92$ & $\begin{array}{l}\text { Cystic fibrosis patient (United Kingdom, } \\
1992 \text { ) }\end{array}$ \\
\hline LMG 13318 & III & xiib, $\mathrm{f}, \mathrm{b}$ & ISL $921 / 92$ & $\begin{array}{l}\text { Cystic fibrosis patient (United Kingdom, } \\
1992 \text { ) }\end{array}$ \\
\hline LMG 13319 & III & xiib, f, b & ISL $857 / 91$ & $\begin{array}{l}\text { Cystic fibrosis patient (United Kingdom, } \\
\text { 1991) }\end{array}$ \\
\hline LMG 13320 & III & xiib, f, b & ISL $863 / 92$ & $\begin{array}{l}\text { Cystic fibrosis patient (United Kingdom, } \\
\text { 1992) }\end{array}$ \\
\hline LMG 13321 & III & xiib, f, b & ISL $860 / 92$ & $\begin{array}{l}\text { Cystic fibrosis patient (United Kingdom, } \\
1992 \text { ) }\end{array}$ \\
\hline LMG 13322 & III & xiib, $f, b$ & ISL 832/91 & $\begin{array}{l}\text { Cystic fibrosis patient (United Kingdom, } \\
\text { 1991) }\end{array}$ \\
\hline LMG 13323 & III & xiib, $f, b$ & ISL $857 / 92$ & $\begin{array}{l}\text { Cystic fibrosis patient (United Kingdom, } \\
\text { 1992) }\end{array}$ \\
\hline LMG 13324 & III & xiib, $f, b$ & ISL $752 / 92$ & $\begin{array}{l}\text { Cystic fibrosis patient (United Kingdom, } \\
\text { 1992) }\end{array}$ \\
\hline LMG 13325 & III & xiib, f, b & ISL $182 / 92$ & $\begin{array}{l}\text { Cystic fibrosis patient (United Kingdom, } \\
\text { 1992) }\end{array}$ \\
\hline LMG 13326 & III & xiib, f, b & ISL $84 / 92$ & $\begin{array}{l}\text { Cystic fibrosis patient (United Kingdom, } \\
\text { 1992) }\end{array}$ \\
\hline LMG 13327 & III & xiib, $f, b$ & ISL $880 / 92$ & $\begin{array}{l}\text { Cystic fibrosis patient (United Kingdom, } \\
1992 \text { ) }\end{array}$ \\
\hline LMG 13328 & III & xiib, $f, b$ & ISL $86 / 92$ & $\begin{array}{l}\text { Cystic fibrosis patient (United Kingdom, } \\
\text { 1992) }\end{array}$ \\
\hline LMG 13329 & III & xiib, f, b & ISL $11 / 92$ & $\begin{array}{l}\text { Cystic fibrosis patient (United Kingdom, } \\
\text { 1992) }\end{array}$ \\
\hline LMG 13330 & III & xiib, f, b & ISL $41 / 92$ & $\begin{array}{l}\text { Cystic fibrosis patient (United Kingdom, } \\
\text { 1992) }\end{array}$ \\
\hline LMG 13331 & III & xiib, f, b & ISL $85 / 92$ & $\begin{array}{l}\text { Cystic fibrosis patient (United Kingdom, } \\
\text { 1992) }\end{array}$ \\
\hline LMG 13332 & III & xiib, f, b & ISL $862 / 92$ & $\begin{array}{l}\text { Cystic fibrosis patient (United Kingdom, } \\
\text { 1992) }\end{array}$ \\
\hline LMG 14088 & III & xiib, f, b & ISL $1 / 93$ & $\begin{array}{l}\text { Cystic fibrosis patient (United Kingdom, } \\
\text { 1993) }\end{array}$ \\
\hline LMG 14089 & III & xiib, f, b & ISL $4 / 93$ & $\begin{array}{l}\text { Cystic fibrosis patient (United Kingdom, } \\
1993 \text { ) }\end{array}$ \\
\hline LMG 14090 & III & xiib, f, b & ISL 5/93 & $\begin{array}{l}\text { Cystic fibrosis patient (United Kingdom, } \\
\text { 1993) }\end{array}$ \\
\hline LMG 14091 & III & xiib, f, b & ISL $54 / 93$ & $\begin{array}{l}\text { Cystic fibrosis patient (United Kingdom, } \\
1993 \text { ) }\end{array}$ \\
\hline LMG 14092 & III & xiib, f, b & ISL $127 / 93$ & $\begin{array}{l}\text { Cystic fibrosis patient (United Kingdom, } \\
1993 \text { ) }\end{array}$ \\
\hline LMG 14093 & III & xiib, f, b & ISL $128 / 93$ & $\begin{array}{l}\text { Cystic fibrosis patient (United Kingdom, } \\
1993 \text { ) }\end{array}$ \\
\hline
\end{tabular}


TABLE 1-Continued

\begin{tabular}{|c|c|c|c|c|}
\hline $\begin{array}{l}\text { Name as received and strain } \\
\text { designation (revised name) })^{a}\end{array}$ & Genomovar & Type of analysis $^{b}$ & $\begin{array}{c}\text { Other strain } \\
\text { designation }(s)^{c}\end{array}$ & Source $^{d}$ \\
\hline LMG 14094 & III & xiib, $f, b$ & ISL $356 / 93$ & $\begin{array}{l}\text { Lung transplant patient (United Kingdom, } \\
\text { 1993) }\end{array}$ \\
\hline LMG 14276 & III & xiib, f, b & NCTC 13009 & Cystic fibrosis patient (Belgium, 1993) \\
\hline LMG 14937 & III & xiib, $f, b$ & & Cystic fibrosis patient (Belgium, 1993) \\
\hline LMG 14941 & III & xiib, $\mathrm{f}, \mathrm{b}$ & & Cystic fibrosis patient (Belgium, 1994) \\
\hline LMG 16656 & III & xiib & $\mathbf{J} 2315$ & $\begin{array}{l}\text { Cystic fibrosis patient (United Kingdom, } \\
\text { 1989), ET12 reference }\end{array}$ \\
\hline LMG 16658 & III & xiib & $\mathrm{C} 1632$ & $\begin{array}{l}\text { Cystic fibrosis patient (United Kingdom), } \\
\text { Newcastle epidemic reference }\end{array}$ \\
\hline LMG 14291 & IV & $v i, f, b$ & & Cystic fibrosis patient (Belgium, 1993) \\
\hline LMG 14294 & IV & $v i, f, b$ & NCTC 13011 & Cystic fibrosis patient (Belgium, 1993) \\
\hline LMG 14295 & IV & $v i, f, b$ & & Cystic fibrosis patient (Belgium, 1993) \\
\hline LMG 14940 & IV & vi, f, b & & Cystic fibrosis patient (Belgium, 1994) \\
\hline LMG 15949 & IV & vi, f, b & & Cystic fibrosis patient (Belgium, 1993) \\
\hline LMG 14939 & & f & & Cystic fibrosis patient (Belgium, 1994) \\
\hline LMG 10824 (B. vietnamiensis) & & vii & & Rice root (Indonesia) \\
\hline LMG 16230 (B. vietnamiensis) & & vii, $f, b$ & CCUG 34416 & Cystic fibrosis patient (Sweden) \\
\hline LMG 16232 (B. vietnamiensis) & & vii, f, b & CCUG 31370 & Cystic fibrosis patient (Sweden) \\
\hline LMG 10823 (B. vietnamiensis) & & viii & & Soil (Indonesia) \\
\hline B. cocovenenans LMG $11626^{\mathrm{T}}$ & & ii, f & LMD $38.18^{\mathrm{T}}$ & Poisoned bongkrek (Java) \\
\hline B. gladioli $\mathrm{LMG} 2216^{\mathrm{T}}$ & & iia, $f, b$ & ATCC $10248^{\mathrm{T}}$ & Gladiolus sp. (United States) \\
\hline B. gladioli LMG 6881 & & iia, $\mathrm{f}$ & NCPPB 1051 & Gladiolus sp. (Zimbabwe) \\
\hline B. gladioli LMG 6882 & & iia, $f$ & NCPPB 1887 & Gladiolus sp. (United States) \\
\hline B. glumae LMG 1277 & & iib, $\mathrm{f}$ & NCPPB 2391 & Oryza sativa (Japan) \\
\hline B. glumae LMG $2196^{\mathrm{T}}$ & & iib, f & NCPPB $2981^{\mathrm{T}}$ & Oryza sativa (Japan) \\
\hline B. glumae LMG 6952 & & iib, $f$ & PDDCC 3728 & Oryza sativa (Japan) \\
\hline B. plantarii LMG $9035^{\mathrm{T}}$ & & iv, $f$ & ICMP $9424^{\mathrm{T}}$ & Oryza sativa (Japan) \\
\hline B. plantarii LMG 10907 & & iv, $\mathrm{f}$ & ICMP 9425 & Soil (Japan) \\
\hline B. plantarii LMG 10908 & & iv, $\mathrm{f}$ & ICMP 9426 & Oryza sativa (Japan) \\
\hline B. vandii $\mathrm{LMG} 16020^{\mathrm{T}}$ & & iv, $f$ & JCM $7957^{\mathrm{T}}$ & Vanda sp. (Japan) \\
\hline B. vietnamiensis LMG 6998 & & vii, $f, b$ & CCUG 7246 & Blood (Sweden, 1978) \\
\hline B. vietnamiensis LMG 10926 & & viii, $f, b$ & TVV 69 & Rice rhizosphere (Vietnam) \\
\hline B. vietnamiensis $\mathrm{LMG} 10929^{\mathrm{T}}$ & & viii, $f, b$ & TVV $75^{\mathrm{T}}$ & Rice rhizosphere (Vietnam) \\
\hline B. vietnamiensis LMG 10930 & & viii, f, b & TVV 116 & Rice rhizosphere (Vietnam) \\
\hline B. vietnamiensis LMG 6999 & & $\mathrm{f}, \mathrm{b}$ & CCUG 9631 & Neck abscess, child (1980) \\
\hline$P$. aeruginosa $\mathrm{LMG} 1242^{\mathrm{T}}$ & & iii & CCEB $481^{\mathrm{T}}$ & \\
\hline P. aeruginosa LMG 5827 & & iii & NCPPB 1966 & Lung \\
\hline$P$. aeruginosa LMG 6395 & & iii & DSM 1117 & Blood culture \\
\hline $\begin{array}{l}\text { P. glathei LMG } 14190^{\mathrm{T}}(B \text {. } \\
\text { glathei) }\end{array}$ & & ix, f & ATCC $29195^{\mathrm{T}}$ & Soil (Germany) \\
\hline $\begin{array}{l}\text { P. glathei LMG } 14932 \text { ( } B \text {. } \\
\text { glathei) }\end{array}$ & & $i x, f$ & ATCC 29198 & Soil (Germany) \\
\hline $\begin{array}{l}\text { P. pyrrocinia } \mathrm{LMG} 14191^{\mathrm{T}}(B . \\
\text { pyrrocinia })\end{array}$ & & $\mathrm{i}, \mathrm{f}$ & ATCC $15958^{\mathrm{T}}$ & Soil \\
\hline R. eutropha LMG $1199^{\mathrm{T}}$ & & & ATCC $17697^{\mathrm{T}}$ & Soil (United States) \\
\hline R. pickettii LMG $5942^{\mathrm{T}}$ & & & CCUG $3318^{\mathrm{T}}$ & Human (United States) \\
\hline R. solanacearum LMG $2299^{\mathrm{T}}$ & & & NCPPB $325^{\mathrm{T}}$ & $\begin{array}{l}\text { Lycopersicon lycopersicum (L.) (United } \\
\text { States) }\end{array}$ \\
\hline
\end{tabular}

$a \mathrm{~T}$, type strain.

${ }^{b} \mathrm{i}$ through xii, protein electrophoresis (the letters refer to positions in the protein electrophoretic dendrogram [Fig. 1]); f, fatty acid analysis; b, biochemical tests.

${ }^{c}$ ATCC, American Type Culture Collection, Rockville, Maryland; CCUG, Culture Collection, University of Göteborg, Department of Clinical Bacteriology, Göteborg, Sweden; JCM, Japan Collection of Microorganisms, Institute of Physical and Chemical Research, Saitama, Japan; LMD, Laboratorium voor Microbiologie, Technische Universiteit, Delft, The Netherlands; LMG, Laboratorium Microbiologie Gent Culture Collection, Universiteit Gent, Ghent, Belgium; NCPPB, National Collection of Plant Pathogenic Bacteria, Harpenden Laboratory, Hertfordshire, United Kingdom; NCTC, National Collection of Type Cultures, London, United Kingdom; PDDCC, Plant Diseases Division Culture Collection, DSIR Mount Albert Research Center, Auckland, New Zealand.

$d$ If known.

ferred to as genomovars I through IV (see below). Strains from electrophoretic clusters $\mathrm{i}$ and $\mathrm{v}$ form a single genotypic subgroup with DNA-DNA binding values above 58\% (genomovar I), while hybridization values towards representatives of other subgroups are between 16 and $45 \%$. Strains of electrophoretic cluster $x$ share DNA-DNA binding values above $82 \%$ (genomovar II), while values towards representatives of other subgroups are between 19 and 54\%. The two strains of electro- phoretic cluster vi have a DNA-DNA binding value of $100 \%$ (genomovar IV), while values towards representatives of other groups are between 23 and 54\%. Strains of electrophoretic clusters xi and xii form a single genotypic subgroup sharing DNA-DNA binding values between 54 and 100\% (genomovar III), while values towards representatives of other subgroups are between 18 and 54\%. Finally, B. vietnamiensis strains of clusters vii and viii share a DNA-DNA binding value of $86 \%$, 


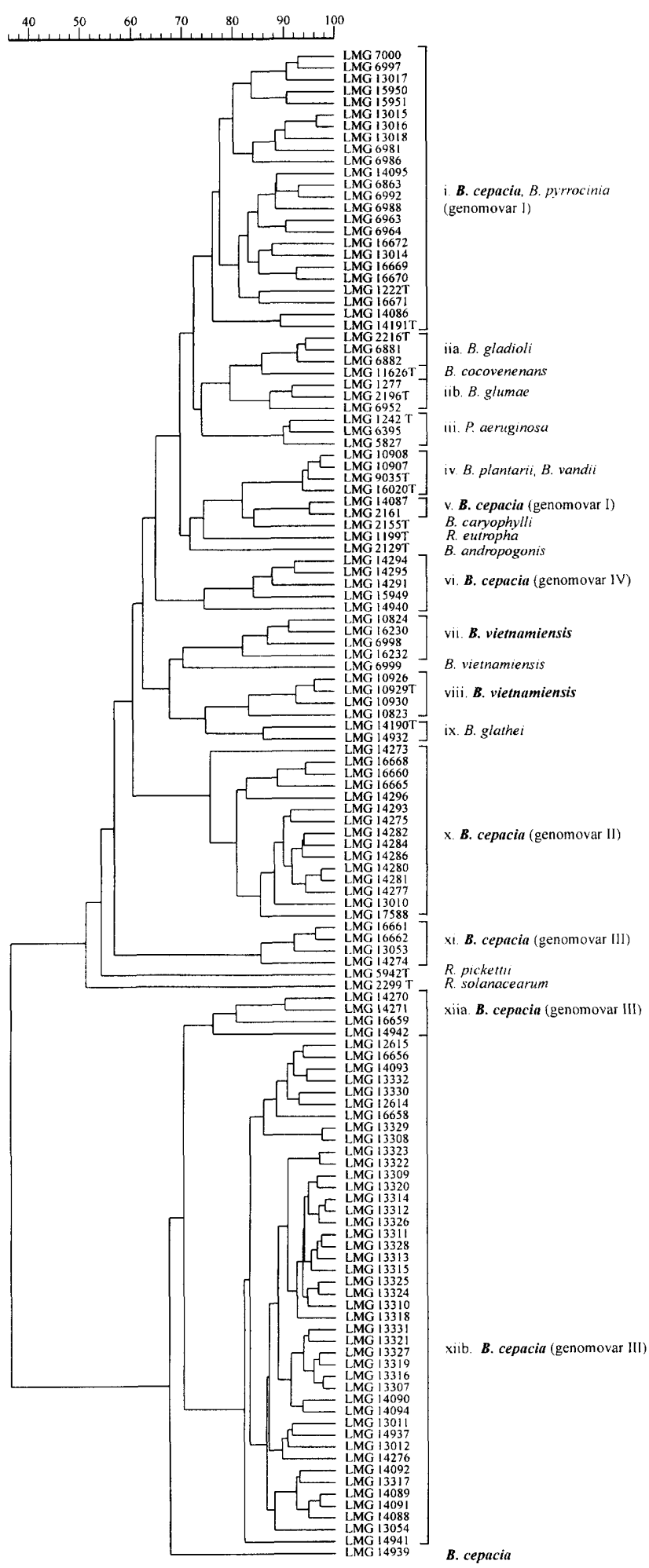

FIG. 1. Dendrogram derived from the unweighted pair group average linkage of correlation coefficients between the protein patterns of all strains studied.

while values towards representatives of other groups are between 16 and $42 \%$.

Strain B. cepacia LMG 14939, with an aberrant position in the protein electrophoretic dendrogram, could not be classified
TABLE 2. $T_{m(e)}$ values of the DNA-rRNA hybrids obtained with 23S rRNA of B. cepacia LMG $1222^{\mathrm{T}}$

\begin{tabular}{|c|c|c|}
\hline DNA source & $\begin{array}{l}\text { Protein electrophoretic } \\
\text { cluster (genomovar) }\end{array}$ & $\begin{array}{l}T_{m(e)} \\
\left({ }^{\circ} \mathrm{C}\right)\end{array}$ \\
\hline B. cepacia $\mathrm{LMG} 1222^{\mathrm{T}}$ & $\mathrm{i}(\mathrm{I})$ & 80.0 \\
\hline B. cepacia LMG 14087 & $\mathrm{v}(\mathrm{I})$ & 80.4 \\
\hline B. cepacia LMG 14294 & vi (IV) & 79.0 \\
\hline B. cepacia LMG 14940 & vi (IV) & 81.6 \\
\hline B. cepacia LMG 13010 & $\mathrm{x}$ (II) & 78.0 \\
\hline B. cepacia $\mathrm{LMG} 14275$ & $\mathrm{x}(\mathrm{II})$ & 79.0 \\
\hline B. cepacia $\mathrm{LMG} 12614$ & xiia (III) & 80.4 \\
\hline B. cepacia LMG 12615 & xiia (III) & 81.0 \\
\hline B. cepacia LMG 13327 & xiia (III) & 80.5 \\
\hline B. vietnamiensis $\mathrm{LMG} 10929^{\mathrm{T}}$ & viii & $81.0^{a}$ \\
\hline B. cocovenenans LMG $11626^{\mathrm{T}}$ & ii & 77.7 \\
\hline B. glumae LMG $2196^{\mathrm{T}}$ & iib & $79.1^{a}$ \\
\hline B. gladioli LMG 6882 & iia & $78.6^{a}$ \\
\hline B. plantarii $\mathrm{LMG} 9035^{\mathrm{T}}$ & iv & $78.4^{a}$ \\
\hline B. andropogonis LMG $2729^{\mathrm{T}}$ & & $76.7^{a}$ \\
\hline B. caryophylli $\mathrm{LMG} 2299^{\mathrm{T}}$ & & $75.5^{a}$ \\
\hline B. glathei LMG $14190^{\mathrm{T}}$ & ix & 76.4 \\
\hline B. pyrrocinia LMG $14191^{\mathrm{T}}$ & $\mathrm{i}$ & 75.5 \\
\hline
\end{tabular}

${ }^{a}$ Data from Gillis et al. (10).

into one of these major genotypic subgroups, although it showed significant hybridization values towards representatives of several subgroups. B. gladioli LMG $2216^{\mathbf{T}}$ and B. cocovenenans LMG $11626^{\mathrm{T}}$ exhibited mostly low hybridization values towards representatives of the different subgroups (below $25 \%$ ). Finally, $P$. pyrrocinia, which belongs to protein electrophoretic subgroup $i$, shares rather high levels of DNA-DNA binding with representatives of several subgroups.

DNA base compositions. All strains examined have DNA base ratios between 67 and 69 mol\% (Table 3).

Cellular fatty acid analysis. Cellular fatty acid analysis was performed on 111 strains (Table 1). The major fatty acid components of all strains examined were 14:0, summed feature 3 , 16:1 $\omega 7 \mathrm{c}, 16: 0,17: 0$ cyclo, 16:0 2OH, 16:0 3OH, summed feature 7, 19:0 cyclo $\omega 8 \mathrm{c}$, and 18:1 2OH. Summed features 3 and 7 comprise fatty acids which cannot be distinguished by the Microbial Identification System. Summed feature 3 contains $16: 1$ iso I or $14: 03 \mathrm{OH}$ or both. It is very likely that for Burkholderia strains summed feature 3 corresponds to $14: 03 \mathrm{OH}$, as this fatty acid has been reported in these strains, whereas 16:1 iso I has not (31). Summed feature 7 contains $18: 1 \omega 7 \mathrm{c}, 18: 1$ $\omega 9 \mathrm{t}$, and 18:1 $\omega 12 \mathrm{t}$, or any combination of these three isomers. Again only one of these fatty acids was reported in Burkholderia strains: $18: 1 \omega 7 \mathrm{c}(31)$.

Average fatty acid compositions were calculated per protein electrophoretic cluster and are given in Table 4 . The overall fatty acid profiles of $B$. cepacia strains belonging to protein electrophoretic clusters i, v (data not included in Table 4), vi, $\mathrm{x}, \mathrm{xi}$, and xii; one $B$. cepacia strain with a separate position in the dendrogram (LMG 14939; data not included in Table 4); and $B$. gladioli were virtually indistinguishable and resembled that of $B$. vietnamiensis strains. Strains of the latter taxon were, however, characterized by higher ratios of $16: 1 \omega 7 \mathrm{c} / 17: 0$ cyclo and 18:1/19:0 cyclo $\omega 8$ c. Overall, $P$. pyrrocinia, $B$. glumae, $B$. andropogonis, $B$. caryophylli, and $P$. glathei were distinguishable from the other taxa examined. $B$. plantarii and $B$. vandii (only a single strain has been described) have very similar fatty acid profiles, although the latter is characterized by a very high percentage of 16:0.

Phenotypic analysis. The biochemical analysis was performed on a total of 74 strains (Table 1) representing all four 


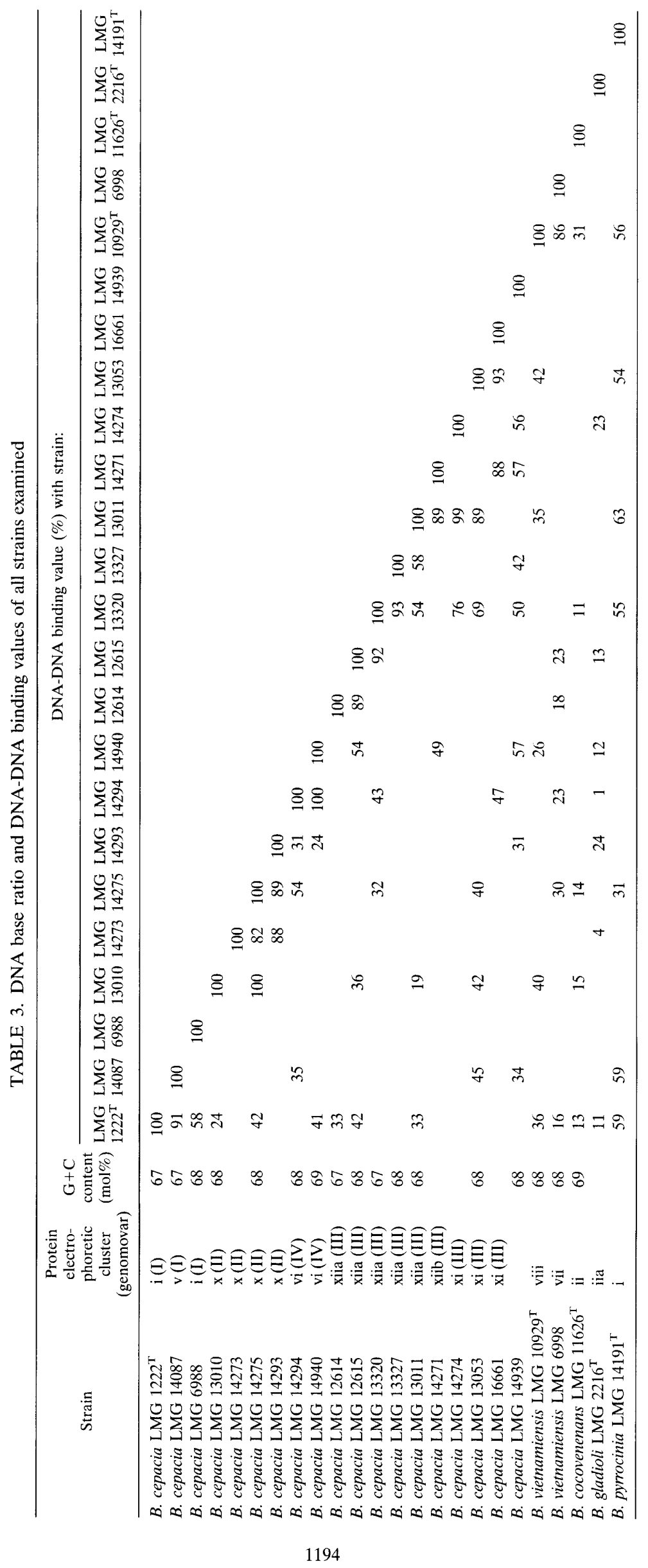




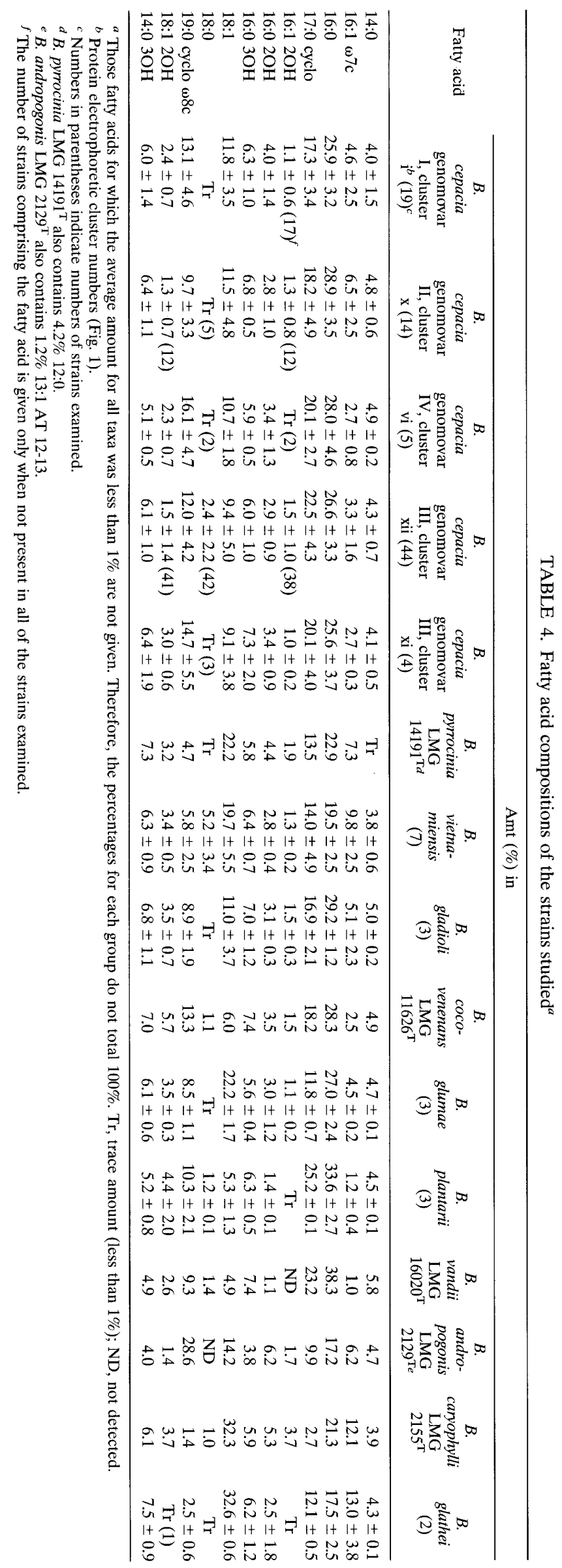

genotypic subgroups within B. cepacia, B. vietnamiensis, and $B$. gladioli. In addition, phenotypic data for $11 \mathrm{~B}$. gladioli strains (these strains are not included in Table 1) isolated from cystic fibrosis patients (5) and identified by DNA-DNA hybridization experiments were included for comparison.

The strains of B. cepacia genomovar III proved very heterogeneous in their phenotypic characters, and a number of nonsaccharolytic variants were observed. The saccharolytic strains produced acid from various numbers of carbohydrates but needed to be divided into two biovars to facilitate practical identification. The nonsaccharolytic strains represent a third biovar. The results for strain LMG 14088 are not presented because this strain gave an oxidative reaction in glucose oxidation-fermentation (OF) medium but produced acid only from glucose in ammonium salt medium; it therefore did not correspond with any of the three biovars recognizable within genomovar III. The most useful characters for differentiating the four genomovars of $B$. cepacia, including the three biovars of genomovar III, and of B. vietnamiensis and B. gladioli are shown in Table 5 .

\section{DISCUSSION}

B. cepacia, traditionally a pathogen in onions (22), has been recognized as an important pathogen in chronic lung disease in patients with cystic fibrosis. A number of observations pointed to heterogeneity among cystic fibrosis isolates of this species and prompted us to collect strains from various sources and from different geographical areas in order to compose a representative collection for a taxonomic study.

Well-characterized reference strains from plants, water, soil, and humans (non-cystic fibrosis patients) and 89 recent isolates identified as $B$. cepacia by means of biochemical tests, cellular fatty acid analysis, or both were included. These 89 strains were isolated primarily from cystic fibrosis patients ( 70 strains isolated from patients in Belgium, Sweden, and the United Kingdom); 2 strains were from lung transplant patients; 4 strains were from non-cystic fibrosis patients; 7 strains were from the hospital environment ( 4 of these were obtained from material or rooms used by cystic fibrosis patients); and 6 strains were isolated from tropical plants or soil.

Representative strains of all of the other Burkholderia species and of $P$. aeruginosa, $R$. solanacearum, $R$. pickettii, and $R$. eutropha were included as references.

Detection of marked heterogeneity among $B$. cepacia strains. SDS-PAGE of whole-cell proteins and computer-assisted comparisons of their protein patterns are often used to compare large numbers of bacteria and to group closely related strains (6). In general, strains with highly similar protein patterns share high DNA-DNA binding values and thus belong to a single genomic species. However, within several species, multiple protein electrophoretic subgroups have been described and are considered electrophoretic types (6). In numerical analyses, electrophoretic types of a single species may form distinct clusters, and therefore the precise relationships between strains belonging to different clusters in a dendrogram (Fig. 1) have to be established by means of DNA-DNA hybridization studies.

In the present numerical comparison of whole-cell protein patterns, $B$. cepacia reference strains and recent isolates constituted eight distinct clusters (Fig. 1), which were clearly distinct from the other reference taxa examined (i.e., various other Burkholderia species, Ralstonia species, and $P$. aeruginosa) (Fig. 1). Six of the B. cepacia reference strains of protein electrophoretic cluster i (LMG $1222^{\mathrm{T}}$, LMG 6981, LMG 6988 , LMG 6963, LMG 6964, and LMG 6997) were previously included in a DNA-DNA hybridization study and shared DNADNA binding values of more than $65 \%(10)$, confirming that 
TABLE 5. Biochemical differentiation of genomovars of B. cepacia, B. vietnamiensis, and B. gladioli

\begin{tabular}{|c|c|c|c|c|c|c|c|c|}
\hline \multirow{4}{*}{ Character } & \multicolumn{8}{|c|}{ Result $^{a}$ for: } \\
\hline & \multicolumn{6}{|c|}{ B. cepacia genomovar } & \multirow{3}{*}{ B. gladioli (12) } & \multirow{3}{*}{ B. vietnamiensis (7) } \\
\hline & \multirow{2}{*}{ I (11) } & \multirow{2}{*}{$\mathrm{II}^{b}(10)$} & \multicolumn{3}{|c|}{ III, biovar } & \multirow{2}{*}{ IV (5) } & & \\
\hline & & & a (8) & $\mathrm{b}(6)$ & $c(25)$ & & & \\
\hline \multicolumn{9}{|l|}{$\begin{array}{l}\text { Acid production in ammonium } \\
\text { salt medium from: }\end{array}$} \\
\hline Glucose & + & + & + & + & - & + & + & + \\
\hline Adonitol & + & + & + & - & - & + & + & - \\
\hline Cellobiose & + & + & + & 5 & - & + & 7 & + \\
\hline Dulcitol & + & + & + & - & - & + & + & + \\
\hline Fructose & + & + & + & - & $2 / 25$ & + & + & + \\
\hline Inositol & + & + & + & 2 & - & + & + & + \\
\hline Lactose & + & + & + & + & - & 4 & - & + \\
\hline Maltose & + & + & 7 & 2 & - & + & - & + \\
\hline Mannitol & + & + & + & - & - & + & + & + \\
\hline Raffinose & $9^{b}$ & - & - & - & - & - & - & 5 \\
\hline Salicin & 10 & 9 & 4 & - & - & - & - & - \\
\hline Sorbitol & + & + & + & - & - & + & + & + \\
\hline Sucrose & 7 & - & + & + & - & - & - & + \\
\hline Xylose & + & + & + & 5 & - & + & + & + \\
\hline $\begin{array}{l}\text { Acid from glucose peptone } \\
\text { water sugar }\end{array}$ & 10 & + & + & 5 & - & + & - & + \\
\hline \multicolumn{9}{|l|}{ Acid from $10 \%(\mathrm{wt} / \mathrm{vol})$} \\
\hline Glucose & + & + & + & + & - & + & 10 & + \\
\hline Lactose & + & + & + & + & - & + & 1 & + \\
\hline $\begin{array}{l}\text { Alkaline reaction in } \mathrm{OF} \\
\text { medium }\end{array}$ & - & - & - & - & $22 / 25$ & - & - & - \\
\hline Casein digestion & 10 & - & 4 & 4 & - & 4 & 11 & - \\
\hline Growth at $42^{\circ} \mathrm{C}$ & - & + & + & 5 & + & - & - & - \\
\hline Growth on cetrimide agar & + & + & + & + & + & + & 2 & + \\
\hline Growth on Simmons citrate & + & + & + & 1 & $4 / 25$ & + & + & + \\
\hline Malonate utilization & + & 9 & 4 & 3 & $1 / 12$ & + & - & + \\
\hline $\begin{array}{l}\text { Melanin-like pigment on } \\
\text { tyrosine agar }\end{array}$ & - & - & 7 & 3 & $1 / 25$ & - & 1 & - \\
\hline Motility at $37^{\circ} \mathrm{C}$ & 3 & + & + & 4 & $16 / 23$ & + & - & + \\
\hline Nitrate reduction & 4 & + & - & 3 & $21 / 25$ & 3 & 5 & 5 \\
\hline Oxidative in OF medium & + & + & + & + & - & + & 11 & + \\
\hline Urease production & 10 & + & 2 & - & $1 / 25$ & 3 & 9 & + \\
\hline
\end{tabular}

${ }^{a}$ Numbers in parentheses are numbers of strains. + , all strains (for $B$. cepacia genomovar III, biovar c, all strains tested) positive; -, all strains (for $B$. cepacia genomovar III, biovar c, all strains tested) negative. The numbers in the field are the numbers of strains giving a positive result; for $B$. cepacia genomovar III, biovar c, the ratios are numbers of strains giving a positive result/numbers of strains tested.

${ }^{b}$ Proposed B. multivorans.

they constitute a single genomic group corresponding to a species as defined by Wayne et al. (41). Two of these strains were included in the present DNA-DNA hybridization study, and a binding value of $58 \%$ was found (Table 3 ). In addition, one of the two B. cepacia strains from cluster v (LMG 14087) also showed a high hybridization value $(91 \%)$ towards the type strain (LMG 1222), indicating that both clusters represent different electrophoretic types of one species (Fig. 1; Table 3).

Similarly, B. cepacia strains of protein electrophoretic clusters xi and xii shared high DNA-DNA hybridization values (average value: $82 \pm 14 \%$; Table 3 ) and thus constitute a single species. Strains of protein electrophoretic clusters vi and $\mathrm{x}$ also formed homogeneous groups. Finally, two $B$. vietnamiensis strains representing electrophoretic clusters vii and viii shared high DNA-DNA hybridization values, confirming a previous report (10).

Hybridizations between representatives of these five genomic groups yielded clearly lower hybridization values, mostly in the range of 20 to $45 \%$ (Table 3 ), and none of the strains shared high DNA-DNA binding values with $B$. gladioli or $B$. cocovenenans, which are close relatives of $B$. cepacia. In the absence of differential classical phenotypic tests, members of this B. cepacia complex were previously referred to as genomovar I (clusters i and v), genomovar II (cluster x), genomovar III (clusters xi and xii), genomovar IV (cluster vi), and $B$. vietnamiensis (clusters vii and viii) $(13,37)$ in accordance with the guidelines and recommendations for the delineation of new species $(36,41)$. These five major genomic groups (including $B$. vietnamiensis) correspond to five distinct species, each with a similar DNA base ratio of approximately $68 \mathrm{~mol} \%$ (Table 3). Finally, the separate position of strain $B$. cepacia LMG 14939 in the protein electrophoretic dendrogram was confirmed by the DNA-DNA hybridization analysis because values between 31 and $57 \%$ were measured towards representatives of $B$. cepacia genomovars I through IV.

Hybridizations between DNA of representative strains of each of these five taxa and rRNA of $B$. cepacia $\mathrm{LMG} 1222^{\mathrm{T}}$ confirm that they all cluster at the top of the $B$. cepacia rRNA 
branch (Table 2). The 16S rRNA sequence of strain LMG 17588 (=ATCC 17616), a typical genomovar II strain, was reported to exhibit more than $98 \%$ similarity with the $16 \mathrm{~S}$ rRNA sequences of $B$. cepacia genomovar I strains (LMG $1222^{\mathrm{T}}$ [ =ATCC $25416^{\mathrm{T}}$ ] and LMG 2161 [=ATCC 17759]) and of $B$. vietnamiensis LMG $10929^{\mathrm{T}}$ (=TVV $75^{\mathrm{T}}$ ) (20). B. gladioli occupies a distinct position, clearly lower on the $B$. cepacia rRNA branch (Table 2) $(10,20)$. B. glumae, B. plantarii, $B$. cocovenenans, and $B$. mallei are its nearest neighbors (Table 2 ) (10).

An analysis of 68 conventional biochemical characteristics (14) yielded a number of tests to differentiate between these genomovars, $B$. vietnamiensis, and B. gladioli, although occasionally strains could be found which do not fit into the expected pattern (Table 5). From these data it appears that particularly $B$. vietnamiensis, $B$. gladioli, and $B$. cepacia genomovar II strains are identifiable. The biochemical differentiation of B. cepacia genomovars I, III, and IV is less clear, and the identification of $B$. cepacia genomovar III can only be achieved by identifying these isolates at the biovar level. Nonsaccharolytic strains of $B$. cepacia (biovar c) are likely to be confused with Alcaligenes or Comamonas strains and were reported earlier by Pitt et al. (24), who demonstrated that saccharolytic and nonsaccharolytic isolates may belong to the same ribotype. Recognition of these nonsaccharolytic strains as $B$. cepacia-like strains was achieved by observing their ability to grow on $B$. cepacia-selective medium, the presence of cytochrome oxidase production, and the absence of arginine hydrolysis (24). Marked phenotypic variability among strains with the same ribotype was also reported by Larsen et al. (18).

There are clearly five genomic species within strains routinely identified as $B$. cepacia. These are mostly distinguishable phenotypically, but occasional atypical characters mean that unequivocal identification is not possible in all cases. At present, we feel there is sufficient biochemical information to propose a species name for genomovar II strains but not for the others. We propose to continue to use the terms $B$. cepacia genomovars I, III, and IV for these taxa pending further study.

$\boldsymbol{P}$. pyrrocinia and $\boldsymbol{P}$. glathei. The taxonomic affiliations of a number of Pseudomonas species have not been fully determined (16). The combined evidence of DNA-rRNA hybridization experiments (Table 2), fatty acid data (the high ratio of the 16:0 $3 \mathrm{OH}$ percentage to the total percentage of 3-hydroxy fatty acids [10]), and DNA-DNA hybridizations indicates that both $P$. pyrrocinia and $P$. glathei belong to the genus Burkholderia $(10)$.

In the present study, $P$. pyrrocinia LMG $14191^{\mathrm{T}}$ could be distinguished from the other taxa examined by its characteristic fatty acid profile but was indistinguishable from $B$. cepacia genomovar I strains in whole-cell protein electrophoresis. Below, we propose to transfer this species into the genus Burkholderia as B. pyrrocinia comb. nov.

In addition, the DNA base ratio (65\% for the type strain [21]) and the general phenotypic and chemotaxonomic profiles of $P$. glathei conform to those of Burkholderia species. Wholecell protein and fatty acid analyses are useful to differentiate this species from other Burkholderia species (Fig. 1; Table 4). Interestingly, the genomes of $P$. glathei strains are composed of multiple chromosomes, a highly unusual characteristic which is present in several other members of the Burkholderia-Ralstonia lineage (20). We conclude that $P$. glathei should be transferred to the genus Burkholderia as B. glathei comb. nov.

Application of whole-cell fatty acid analysis for the differentiation of Burkholderia species. Several investigators examined the whole-cell fatty acid components of Burkholderia strains; this technique is often used for the identification of $B$. cepacia strains. When comparing the results of different fatty acid analyses, it is important to realize that the quantitative composition may be strongly influenced by various factors including growth medium, time, and temperature. Urakami et al, (35) examined several Burkholderia species and could not detect differences in their fatty acid patterns, whereas Yabuuchi et al. (43) reported clear differences between the type strains of several species. In both studies, only one or two reference strains per species were included. Stead (31) examined several strains per species and presented the average value and standard deviation per taxon. B. cepacia, B. gladioli, and some of the $B$. glumae strains had indistinguishable profiles; other Burkholderia species were readily differentiated (31). In our study (Table 4), the B. cepacia genomovars and B. gladioli were indistinguishable. Only a single $B$. cocovenenans strain was examined, and its pattern was very similar to those of the $B$. cepacia and $B$. gladioli strains. In addition, the $B$. vandii and $B$. plantarii strains were characterized by a lower percentage of $18: 1$, a higher percentage of $16: 0$, and a lower ratio of $16: 1$ $\omega 7 \mathrm{c} / 17: 0$ cyclo compared to $B$. cepacia and $B$. gladioli strains. However, considering all standard deviations, it is questionable if these differences will suffice to identify unknown field isolates to the species level. In contrast, B. vietnamiensis, B. glumae, and the other species examined all appeared distinguishable.

Genome structure and implications in taxonomy. The $B$. cepacia genome exhibits a number of salient features. A remarkable variability in genome size and organization has been reported, and a considerable number of insertion sequences have been described $(4,20,27)$. A comparison of the $16 \mathrm{~S}$ rRNA sequences explained some of this variability, as one of the strains examined was shown to be a close neighbor of $B$. caryophylli, not of $B$. cepacia (20). Several other strains (including $B$. cepacia genomovars I and II and $B$. vietnamiensis strains) were reported to share more than $98 \%$ of their $16 \mathrm{~S}$ rRNA sequences. This is obviously not a unique situation: biochemically similar groups with more than $98 \% 16 \mathrm{~S}$ rRNA sequence similarity and a low level of DNA-DNA hybridization are present in many genera and are considered different species if sufficient differential phenotypic characteristics are available $(29,36,41)$. However, the genomes of $B$. cepacia and related species (including $B$. glumae, $R$. pickettii, $R$. eutropha, $R$. solanacearum, and B. glathei) are unusual because of their organization in multiple (two to four) chromosomes or replicons. The cumulative molecular size of these individual DNA circles of presumed $B$. cepacia strains varies between 4.6 and $8.1 \mathrm{Mb}$ (20). The genomic molecular sizes for genomovar I strains LMG $1222^{\mathrm{T}}\left(=\right.$ ATCC $\left.25415^{\mathrm{T}}\right)$ and LMG $2161 \quad(=\mathrm{ATCC}$ 17759 ) are 8.0 and $7.9 \mathrm{Mb}$, respectively, while a value of $6.8 \mathrm{Mb}$ was reported for a genomovar II strain (LMG 17588 [ = ATCC 17616]). At present, it has not been established that this molecular size is more or less constant within the genomovars, although this is what one would expect from a "normal" bacterial species. It is tempting to speculate on the source of this biological diversity. Could these genomovars have emerged out of each other by the loss or accumulation of large genomic fragments or even entire replicons? The present evidence derived from comparative study of DNA fragments generated by macrorestriction fragment analysis does not support this hypothesis (20), as there is a total lack of common fragments between the patterns of different strains examined. All four $B$. cepacia genomovars and $B$. vietnamiensis have the same DNA base ratio (Table 3 ), so it seems unlikely that some of these replicons are derived from remotely related species. Whether or not these genomovars emerged out of each other, they are composed of different isolates, mostly derived from various 
sources and from different geographical areas. Whatever the underlying evolutionary events, each of the genomovars comprises several distinct strains, as determined by a variety of DNA-based typing methods (38), having a high level of overall DNA homology and a common set of proteins as do the majority of bacterial species.

Practical implications. Because of the increased interest in $B$. cepacia, several studies focused on strategies for the identification of $B$. cepacia strains. Cellular fatty acid analysis, different commercial systems based on phenotypic tests, DNA probes derived from $16 \mathrm{~S}$ and $23 \mathrm{~S}$ rRNA genes, and growth on selective media $(17,19,28)$ were all evaluated and were often shown to be either not very sensitive or not very specific or both. From the data shown in the present study, it is obvious that a reevaluation of the specificity and sensitivity of each of these strategies is warranted.

The heterogeneity among presumed $B$. cepacia strains may have other important practical implications. Cystic fibrosis patients are particularly at risk for $B$. cepacia infections, and draconian segregation policies for $B$. cepacia-colonized patients have reduced but not entirely eliminated the incidence of colonization in many centers (12). There is also an unexplained variability in the outcome of $B$. cepacia infection in cystic fibrosis patients. Some patients succumb to $B$. cepacia syndrome (15) and die within weeks or months after colonization; others remain stable for years. Although the outcome of infection by the same genetic clone, as determined by highly discriminatory typing methods, may vary among patients, part of these differences could be explained by differences among individual $B$. cepacia strains. Closely related species may have the same pathogenic potential, and it is likely that virulence factors will be exchanged more readily between closely related taxa. However, this is not necessarily the case, as demonstrated by $B$. mallei and B. pseudomallei, or additionally, by Bordetella pertussis and Bordetella bronchiseptica. These taxa share a high level of DNA-DNA binding ( $>80 \%$; i.e., considerably higher than the binding levels among the different $B$. cepacia genomovars) but have very different pathogenic potentials. The intercontinental Edinburgh-Toronto strain (the so-called ET12 epidemic lineage represented by strain LMG $16656[=\mathrm{J} 2315]$ $[11,13,32])$ responsible for many deaths among cystic fibrosis patients belongs to genomovar III. Other epidemic strains which caused multiple deaths among cystic fibrosis patients in the United Kingdom (the Newcastle and Manchester outbreaks [28], represented by strains LMG 16658 and LMG 16659, respectively) also belong to genomovar III. However, another epidemic strain affecting mainly healthy children belongs to genomovar II (the Glasgow outbreak [42], represented by strain LMG 16660 ).

In the present study, the majority of "B. cepacia" strains isolated from cystic fibrosis patients belong to genomovar III (Table 1) (49 strains out of a total of 70 cystic fibrosis isolates) and genomovar II (12 strains), while few isolates belong to genomovars I (1 strain) and IV (5 strains) or B. vietnamiensis (2 strains) (one strain, LMG 14939, occupied a separate taxonomic position). It should be emphasized that the distribution of genomovars in the cystic fibrosis patient community (or other niches) has not been systematically determined and that the pathogenic significance of the different genomovars is not known.

The problem is given an extra dimension by agricultural microbiologists who continue to develop $B$. cepacia as a biocontrol agent to use its antifungal activity and its nutritional versatility for bioremediation. Clearly, the roles and pathogenic significance of $B$. cepacia genomovars, B. vietnamiensis, and $B$. gladioli, all of which occur in cystic fibrosis patients, must be established in order to propose scientifically founded policies for each of the problems.

Conclusions. We performed an extensive phenotypic and genotypic analysis of 128 Burkholderia strains and related bacteria. Our data show that strains identified as B. cepacia by means of biochemical tests or cellular fatty acid analysis may belong to at least five different species. It has been proposed to refer to this group of five phenotypically similar species as the $B$. cepacia complex (third meeting of the International Burkholderia cepacia Working Group, May 10 to 11, 1997, Victoria, British Columbia, Canada). B. cepacia genomovar II, for which we propose the name $B$. multivorans below, and $B$. vietnamiensis are identifiable by means of classical phenotypic tests (Table 5), whole-cell protein electrophoresis (Fig. 1), and DNA-DNA hybridization experiments (Table 3 ); B. cepacia genomovars I, III, and IV can be differentiated by means of whole-organism protein electrophoresis (Fig. 1) and DNA-DNA hybridization experiments (Table 3). Representative strains of genomovars II (LMG 13010 [=NCTC 13007], LMG 14273, and LMG 14293), III (LMG 12614 [=NCTC 13010] [biovar c]), LMG 12615 [=NCTC 13008] [biovar a], LMG 14276 [=NCTC 13009] [biovar b], LMG 14271 [biovar status not determined], and LMG 14274 [biovar status not determined]), and IV (LMG 14294 [=NCTC 13011] and LMG 14940) have been deposited in the BCCM/LMG and NCTC culture collections.

"Pseudomonas multivorans" (30) was shown to represent the same taxon as Pseudomonas cepacia, and therefore this name lost standing in bacterial nomenclature. We chose to use this specific epithet for the $B$. cepacia genomovar II strains because it both highlights the nutritional versatility of these organisms and implicitly refers to their relatedness to $B$. cepacia strains.

Description of Burkholderia multivorans sp. nov. Burkholderia multivorans (mul.ti'vo.rans L. adj. multus, much; L. part. adj. vorans, devouring, digesting; M.L. part. adj. multivorans digesting many compounds) cells are 1.0 to $2.0 \mu \mathrm{m}$ long and 0.6 to $0.9 \mu \mathrm{m}$ wide and are motile at room temperature and at $37^{\circ} \mathrm{C}$. Growth is observed at 37 and $42^{\circ} \mathrm{C}$, not at $5^{\circ} \mathrm{C}$; some strains grow at room temperature. So far, no pigmented strains have been detected and no melanin-like pigment is produced on tyrosine agar. Growth on MacConkey agar and Simmons citrate agar. No alkaline reaction in Hugh and Leifson OF medium. Oxidative in OF medium. Alkaline reaction on Christensen's citrate agar. Growth in the presence of cetrimide. No growth in the presence of $0.4 \%$ selenite. Tolerance to $\mathrm{KCN}$ is strain dependent. No fluorescence on King's B medium. Tyrosine, Tween 20 , and Tween 80 are hydrolyzed. Urease, catalase, oxidase, beta-galactosidase ( 9 of 10 strains tested), and lecithinase activities are present. Nitrate is reduced, not nitrite. No indole, hydrogen sulfide, or 3-ketolactose production; no liquefaction of gelatin; no hydrolysis of esculin, casein, or starch; and no DNase activity. No lysine or ornithine decarboxylase, arginine dihydrolase, or arginine desimidase activity. No oxidation of gluconate. No production of phenylpyruvic acid. Most strains utilize malonate ( 9 of 10 tested). Poly- $\beta$ hydroxybutyrate is utilized and present as inclusion granules. Acid production from glucose peptone water sugar but no gas. Acid production from $10 \%$ (wt/vol) glucose and from $10 \%$ (wt/vol) lactose. Acid is produced from the following sugars in ammonium salt medium: glucose, adonitol, arabinose, cellobiose, dulcitol, glycerol, inositol, lactose, maltose, mannitol, salicin (9 of 10 strains tested), sorbitol, trehalose, xylose, fructose, and ethanol ( 8 of 10 strains tested), but not from raffinose, rhamnose, or sucrose.

The DNA base ratio is 68 to 69 mol\%. Major fatty acid components are 14:0 (about 4.8\%), 14:0 30H (about 6.4\%), 16:1 $\omega 7 \mathrm{c}$ (about 6.5\%), 16:0 (about 28.9\%), 17:0 cyclo (about 
$18.2 \%$ ), 16:0 30H (about 6.8\%), 18:1 (about 11.5\%), and 19:0 cyclo $\omega 8 \mathrm{c}$ (about $9.7 \%$ ).

So far, B. multivorans strains have been primarily isolated from sputum of cystic fibrosis patients but have also been isolated from an infection in a non-cystic fibrosis patient, from the hospital environment, and from soil. Pathogenicity is unknown. The type strain is LMG 13010, which was isolated in Brussels from the sputum of a cystic fibrosis patient. Its DNA base ratio is $68 \mathrm{~mol} \%$, and its phenotypic characteristics are the same as those described above for the species. Characteristics differentiating $B$. multivorans from related taxa are summarized in Tables 4 and 5 .

Description of Burkholderia glathei (Zolg and Ottow 1975) comb. nov. The description of $B$. glathei is as given in Bergey's Manual of Systematic Bacteriology (21). In addition, the prominent fatty acids of $B$. glathei strains are 14:0 (about 4.3\%), 14:0 3OH (about 7.5\%), 16:1 $\omega 7 \mathrm{c}$ (about 13.0\%), 16:0 (about $17.5 \%$ ), 17:0 cyclo (about 12.1\%), 16:0 3OH (about $6.2 \%$ ), and 18:1 (about 32.6\%). The type strain is LMG 14190.

Description of Burkholderia pyrrocinia (Imananka, Kousaka, Tamura, and Arima 1965) comb. nov. The description of $B$. pyrrocinia is as given in Bergey's Manual of Systematic Bacteriology (21). In addition, the prominent fatty acids of $B$. pyrrocinia LMG $14191^{\mathrm{T}}$ are 12:0 (4.2\%), 14:0 3OH (7.3\%), 16:1 $\omega 7 \mathrm{c}(7.3 \%), 16: 0(22.9 \%), 17: 0$ cyclo (about 13.5\%), 16:0 2OH (4.4\%), 16:0 30H (5.8\%), 18:1 (22.2\%), and 19:0 cyclo $\omega 8 \mathrm{c}$ (4.7\%). The type strain is LMG 14191.

\section{ACKNOWLEDGMENTS}

We are extremely grateful to P. Borman, M. Ganner, F. Depoorter, D. Dewettinck, and U. Torck for their able technical assistance.

P.V. is indebted to the Fund for Scientific Research-Flanders (Belgium) for a position as a postdoctoral fellow. T.C. acknowledges the support received from the Vlaams Instituut voor Bevordering van Wetenschappelijk-technologisch Onderzoek in de Industrie (Belgium) in the form of a bursary for advanced study. K.K. and M.G. are indebted to the Fund for Medical Scientific Research, Belgium, for research and personnel grants. We acknowledge the financial support by the Cystic Fibrosis Trust (United Kingdom) (grant RS15) and the European Community (contracts BIOT-CT91-0294 and BIO2-CT943098). Part of the research was performed in the framework of BCCM and was cofinanced by the Prime Minister's Services-Federal Office for Scientific, Technical, and Cultural Affairs (Belgium).

\section{REFERENCES}

1. Anonymous. 1992. Pseudomonas cepacia-more than a harmless commensal? Lancet 339:1385-1386. (Editorial.)

2. Bevivino, A., S. Tabacchioni, L. Chiarini, M. V. Carusi, M. Del Gallo, and P. Visca. 1994. Phenotypic comparison between rhizosphere and clinical isolates of Burkholderia cepacia. Microbiology 140:1069-1077.

3. Butler, S. L., C. J. Doherty, J. E. Hughes, J. W. Nelson, and J. R. W. Govan. 1995. Burkholderia cepacia and cystic fibrosis: do natural environments present a potential hazard? J. Clin. Microbiol. 33:1001-1004.

4. Cheng, H.-P., and T. Lessie. 1994. Multiple replicons constituting the genome of Pseudomonas cepacia 17616. J. Bacteriol. 176:4034-4042.

5. Christenson, J. C., D. F. Welch, G. Mukwaya, M. J. Muszynski, R. E. Weaver, and D. J. Brenner. 1989. Recovery of Pseudomonas gladioli from respiratory tract specimens of patients with cystic fibrosis. J. Clin. Microbiol. 27:270-273.

6. Costas, M. 1992. Classification, identification, and typing of bacteria by the analysis of their one-dimensional polyacrylamide gel electrophoretic protein patterns, p. 351-408. In A. Chambrach, M. J. Dunn, and B. J. Radola (ed.) Advances in electrophoresis, vol. 5. VCH Verlagsgesellschaft, Weinheim, Germany.

7. De Ley, J. 1970. Reexamination of the association between melting point, buoyant density, and chemical base composition of deoxyribonucleic acid. J. Bacteriol. 101:738-754.

8. De Ley, J., H. Cattoir, and A. Reynaerts. 1970. The quantitative measurement of DNA hybridization from renaturation rates. Eur. J. Biochem. 12: 133-142.

9. De Vos, P., and J. De Ley. 1983. Intra- and intergeneric similarities of Pseudomonas and Xanthomonas ribosomal ribonucleic acid cistrons. Int. J. Syst. Bacteriol. 33:487-509.
10. Gillis, M., T. V. Van, R. Bardin, M. Goor, P. Hebbar, A. Willems, P. Segers, K. Kersters, T. Heulin, and M. P. Fernandez. 1995. Polyphasic taxonomy in the genus Burkholderia leading to an emended description of the genus and proposition of Burkholderia vietnamiensis sp. nov. for $\mathrm{N}_{2}$-fixing isolates from rice in Vietnam. Int. J. Syst. Bacteriol. 45:274-289.

11. Govan, J. R. W., P. H. Brown, J. Maddison, C. J. Doherty, J. W. Nelson, M. Dodd, A. P. Greening, and A. K. Webb. 1993. Evidence for transmission of Pseudomonas cepacia by social contact in cystic fibrosis. Lancet 342:15-19.

12. Govan, J. R. W., and V. Deretic. 1996. Microbial pathogenesis in cystic fibrosis: mucoid Pseudomonas aeruginosa and Burkholderia cepacia. Microbiol. Rev. 60:539-574.

13. Govan, J. R. W., J. Hughes, and P. Vandamme. 1996. Burkholderia cepacia: medical, taxonomic and ecological issues. J. Med. Microbiol. 45:395-407.

14. Holmes, B., S. P. Lapage, and H. Malnick. 1975. Strains of Pseudomonas putrefaciens from clinical material. J. Clin. Pathol. 28:149-155.

15. Isles, A., I. Maclusky, M. Corey, R. Gold, C. Prober, P. Fleming, and H. Levison. 1984. Pseudomonas cepacia infection in cystic fibrosis: an emerging problem. J. Pediatr. 104:206-210.

16. Kersters, K., W. Ludwig, M. Vancanneyt, P. De Vos, M. Gillis, and K.-H. Schleifer. 1996. Recent changes in the classification of the pseudomonads: an overview. Syst. Appl. Microbiol. 19:465-477.

17. Kiska, D. L., A. Kerr, M. C. Jones, J. A. Caracciolo, B. Eskridge, M. Jordan, S. Miller, D. Hughes, N. King, and P. Gilligan. 1996. Accuracy of four commercial systems for identification of Burkholderia cepacia and other gram-negative nonfermenting bacilli recovered from patients with cystic fibrosis. J. Clin. Microbiol. 34:886-891.

18. Larsen, G. Y., T. L. Stull, and J. L. Burns. 1993. Marked phenotypic variability in Pseudomonas cepacia isolated from a patient with cystic fibrosis. J. Clin. Microbiol. 31:788-792.

19. Leff, L. G., R. M. Kernan, J. V. McArthur, and L. J. Shimkets. 1995. Identification of aquatic Burkholderia (Pseudomonas) cepacia by hybridization with species-specific rRNA gene probes. Appl. Environ. Microbiol. 61:1634-1636.

20. Lessie, T. G., W. Hendrickson, B. D. Manning, and R. Devereux. 1996. Genomic complexity and plasticity of Burkholderia cepacia. FEMS Microbiol. Lett. 144:117-128.

21. Palleroni, N. J. 1984. Genus I. Pseudomonas Migula $1894,237^{\mathrm{AL}}$ (nom. cons. opin. 5, Jud. Comm. 1952, 237), p. 141-199. In N. R. Krieg and J. G. Holt (ed.), Bergey's manual of systematic bacteriology, vol. I. Williams \& Wilkins, Baltimore, Md.

22. Palleroni, N. J., and B. Holmes. 1981. Pseudomonas cepacia sp. nov., nom. rev. Int. J. Syst. Bacteriol. 31:479-481.

23. Palleroni, N. J., R. Kunisawa, R. Contopoulou, and M. Doudoroff. 1973. Nucleic acid homologies in the genus Pseudomonas. Int. J. Syst. Bacteriol. 23:333-339.

24. Pitt, T. L., M. E. Kaufmann, P. S. Patel, L. C. A. Benge, S. Gaskin, and D. M. Livermore. 1996. Type characterization and antibiotic susceptibility of Burkholderia (Pseudomonas) cepacia isolates from patients with cystic fibrosis in the United Kingdom and the Republic of Ireland. J. Med. Microbiol. 44: 203-210.

25. Pot, B., P. Vandamme, and K. Kersters. 1994. Analysis of electrophoretic whole-organism protein fingerprinting, p. 493-521. In M. Goodfellow and A. G. O'Donnell (ed.), Modern microbial methods. Chemical methods in bacterial systematics. J. Wiley and Sons, Chichester, United Kingdom.

26. Revets, H., P. Vandamme, A. Van Zeebroeck, K. De Boeck, M. J. Struelens, J. Verhaegen, J. P. Ursi, G. Verschraegen, H. Franckx, A. Malfroot, I. Dab, and S. Lauwers. 1996. Burkholderia (Pseudomonas) cepacia and cystic fibrosis: the epidemiology in Belgium. Acta Clin. Belg. 51:222-230.

27. Rodley, P. D., U. Römling, and B. Tümmler. 1995. A physical map of the Burkholderia cepacia type strain. Mol. Microbiol. 17:57-67.

28. Simpson, I. N., J. Finlay, D. J. Winstanley, N. Dewhurst, J. W. Nelson, S. L. Butler, and J. R. W. Govan. 1994. Multi-resistance isolates possessing characteristics of both Burkholderia (Pseudomonas) cepacia and Burkholderia gladioli from patients with cystic fibrosis. J. Antimicrob. Chemother. 34:353-361.

29. Stackebrandt, E., and B. M. Goebel. 1994. Taxonomic note: a place for DNA-DNA reassociation and 16S rRNA sequence analysis in the present species definition in bacteriology. Int. J. Syst. Bacteriol. 44:846-849.

30. Stanier, R. Y., N. J. Palleroni, and M. Doudoroff. 1966. The aerobic pseudomonads: a taxonomic study. J. Gen. Microbiol. 43:159-271.

31. Stead, D. E. 1992. Grouping of plant-pathogenic bacteria and some other Pseudomonas spp. by using cellular fatty acid profiles. Int. J. Syst. Bacteriol. 42:281-295.

32. Sun, L., R.-Z. Jiang, S. Steinbach, A. Holmes, C. Campanelli, J. Forstner, Y. Tan, M. Riley, and R. Goldstein. 1995. The emergence of a highly transmissible lineage of $\mathrm{Cbl}^{+}$Pseudomonas (Burkholderia) cepacia causing CF center epidemics in North America and Britain. Nat. Med. 1:661-666.

33. Tabacchioni, S., P. Visca, L. Chiarini, A. Bevivino, C. Di Serio, S. Fancelli, and R. Fani. 1995. Molecular characterization of rhizosphere and clinical isolates of Burkholderia cepacia. Res. Microbiol. 146:531-542.

34. Tyler, S. D., C. A. Strathdee, K. R. Rozee, and W. M. Johnson. 1995 Oligonucleotide primers designed to differentiate pathogenic pseudomonads on the basis of the sequencing of genes coding for $16 \mathrm{~S}-23 \mathrm{~S}$ rRNA internal 
transcribed spacers. Clin. Diagn. Lab. Immunol. 2:448-453.

35. Urakami, T. C. Ito-Yoshida, H. Araki, T. Kijima, K.-I. Suzuki, and K. Komagata. 1994. Transfer of Pseudomonas plantarii and Pseudomonas glumae to Burkholderia as Burkholderia spp. and description of Burkholderia vandii sp. nov. Int. J. Syst. Bacteriol. 44:235-245.

36. Ursing, J. B., R. A. Rosselló-Mora, E. García-Valdés, and J. Lalucat. 1995. Taxonomic note: a pragmatic approach to the nomenclature of phenotypically similar genomic groups. Int. J. Syst. Bacteriol. 45:604.

37. Vandamme, P. 1995. Emerging new "Pseudomonas" species in cystic fibrosis. Presented at the 20th European Cystic Fibrosis Conference, Brussels, Belgium.

38. Vandamme, P. Unpublished data.

39. Vandamme, P., M. Vancanneyt, B. Pot, L. Mels, B. Hoste, D. Dewettinck, L. Vlaes, C. Van Den Borre, R. Higgins, J. Hommez, K. Kersters, J.-P. Butzler, and H. Goossens. 1992. Polyphasic taxonomic study of the emended genus Arcobacter with Arcobacter butzleri comb. nov. and Arcobacter skirrowii sp. nov., an aerotolerant bacterium isolated from veterinary specimens. Int. J. Syst. Bacteriol. 42:344-356.

40. Van Landschoot, A., and J. De Ley. 1983. Intra- and intergeneric similarities of the rRNA cistrons of Alteromonas, Marinomonas (gen. nov.) and some other Gram-negative bacteria. J. Gen. Microbiol. 129:3057-3074.

41. Wayne, L. G., D. J. Brenner, R. R. Colwell, P. A. D. Grimont, P. Kandler, M. I. Krichevsky, L. H. Moore, W. E. C. Moore, R. G. E. Murray, E. Stackebrandt, M. P. Starr, and H. G. Trüper. 1987. Report of the ad hoc committee on reconciliation of approaches to bacterial systematics. Int. J. Syst. Bacteriol. 37:463-464.

42. Whiteford, M. L., J. D. Wilkinson, J. H. McColl, F. M. Conlon, J. R. Michie, T. J. Evans, and J. Y. Paton. 1995. Outcome of Burkholderia (Pseudomonas) cepacia colonisation in children with cystic fibrosis following a hospital outbreak. Thorax 50:1194-1198.

43. Yabuuchi, E., Y. Kosako, H. Oyaizu, I. Yano, H. Hotta, Y. Hashimoto, T. Ezaki, and M. Arakawa. 1992. Proposal of Burkholderia gen. nov. and transfer of seven species of the genus Pseudomonas homology group II to the new genus, with the type species Burkholderia cepacia (Palleroni and Holmes 1981) comb. nov. Microbiol. Immunol. 36:1251-1275.

44. Yabuuchi, E., Y. Kosako, I. Yano, H. Hotta, and Y. Nishiuchi. 1995. Transfer of two Burkholderia and an Alcaligenes species to Ralstonia gen. nov.: proposal of Ralstonia pickettii (Ralston, Palleroni and Doudoroff 1973) comb. nov., Ralstonia solanacearum (Smith 1896) comb. nov. and Ralstonia eutropha (Davis 1969) comb. nov. Microbiol. Immunol. 39:897-904.

45. Yohalem, D. S., and J. W. Lorbeer. 1994. Multilocus isoenzyme diversity among strains of Pseudomonas cepacia isolated from decayed onions, soils, and clinical sources. Syst. Appl. Microbiol. 17:116-124.

46. Zhao, N., C. Qu, E. Wang, and W. Chen. 1995. Phylogenetic evidence for the transfer of Pseudomonas cocovenenans (van Damme et al. 1960) to the genus Burkholderia as Burkholderia cocovenenans (van Damme et al. 1960) comb. nov. Int. J. Syst. Bacteriol. 45:600-603. 